PROCEEDINGS OF THE

AMERICAN MATHEMATICAL SOCIETY

Volume 128, Number 1, Pages 195-201

S 0002-9939(99)04991-6

Article electronically published on May 27, 1999

\title{
INTEGRABILITY OF SUPERHARMONIC FUNCTIONS IN A JOHN DOMAIN
}

\author{
HIROAKI AIKAWA
}

(Communicated by Albert Baernstein II)

\begin{abstract}
The integrability of positive superharmonic functions on a bounded fat John domain is established. No exterior conditions are assumed. For a general bounded John domain the $L^{p}$-integrability is proved with the estimate of $p$ in terms of the John constant.
\end{abstract}

\section{INTRODUCTION}

Let $D$ be a bounded domain in $\mathbb{R}^{n}$ with $n \geq 2$. By $S^{+}(D)$ we denote the family of all positive superharmonic functions in $D$. Armitage [5], [6] proved that $S^{+}(D) \subset L^{p}(D)$ for $0<p<n /(n-1)$, provided $D$ is smooth. This result was extended by Maeda-Suzuki [11] to a Lipschitz domain. They gave an estimate of $p$ in terms of Lipschitz constant. Their estimate has the correct asymptotic behavior: $p \rightarrow n /(n-1)$ as the Lipschitz constant tends to 0 . As a result they showed that $S^{+}(D) \subset L^{p}(D)$ for $0<p<n /(n-1)$, provided $D$ is a $C^{1}$ domain. Masumoto [12], [13] succeeded in obtaining the sharp value of $p$ for planar domains bounded by finitely many Jordan curves. For the higher dimensional case Aikawa [1] gave the sharp value of $p$ for Lipschitz domains with the aid of the coarea formula and the boundary Harnack principle.

On the other hand, Stegenga-Ullrich [16] treated very non-smooth domains, such as John domains and domains satisfying the quasihyperbolic boundary condition $[7,3.6]$, which are called "Hölder domains" by Smith-Stegenga [15]. Let $\delta_{D}(x)=$ $\operatorname{dist}(x, \partial D)$ and $x_{0} \in D$. We say that $D$ is a John domain with John constant $c_{J}>0$ if each $x \in D$ can be joined to $x_{0}$ by a rectifiable curve $\gamma$ such that

$$
\delta_{D}(\xi) \geq c_{J} \ell(\gamma(x, \xi)) \text { for all } \xi \in \gamma,
$$

where $\gamma(x, \xi)$ is the subarc of $\gamma$ from $x$ to $\xi$ and $\ell(\gamma(x, \xi))$ is the length of $\gamma(x, \xi)$. A John domain may be visualized as a domain satisfying a twisted cone condition. The quasi-hyperbolic metric $k_{D}\left(x_{1}, x_{2}\right)$ is defined by

$$
k_{D}\left(x_{1}, x_{2}\right)=\inf _{\gamma} \int_{\gamma} \frac{d s}{\delta_{D}(x)}
$$

Received by the editors March 17, 1998.

1991 Mathematics Subject Classification. Primary 31B05.

This work was supported in part by Grant-in-Aid for Scientific Research (B) (No. 09440062), Japanese Ministry of Education, Science and Culture. 
where the infimum is taken over all rectifiable arcs $\gamma$ joining $x_{1}$ to $x_{2}$ in $D$. We say that $D$ satisfies a quasi-hyperbolic boundary condition if there are positive constants $A_{1}$ and $A_{2}$ such that

$$
k_{D}\left(x, x_{0}\right) \leq A_{1} \log \left(\frac{1}{\delta_{D}(x)}\right)+A_{2} \quad \text { for all } x \in D .
$$

Smith-Stegenga [15] called a domain satisfying the quasihyperbolic boundary condition a Hölder domain. It is easy to see that a John domain satisfies the quasihyperbolic boundary condition (see [7, Lemma 3.11]). Stegenga-Ullrich [16] proved that $S^{+}(D) \subset L^{p}(D)$ with small $p>0$ for a domain satisfying the quasihyperbolic boundary condition. Lindqvist [10] extends the result to positive supersolutions of certain nonlinear elliptic equations, such as the $p$-Laplace equation. Gotoh [8] also studies $L^{p}$-integrability. Unfortunately, their $p>0$ is very small and it does not seem that $p \geq 1$ is obtained by their methods.

The main aim of the present paper is to show that $S^{+}(D) \subset L^{1}(D)$ for a "fat" John domain.

Theorem 1. Let $D$ be a bounded John domain with John constant $c_{J} \geq 1-2^{-n-1}$. Then $S^{+}(D) \subset L^{1}(D)$.

The above bound $1-2^{-n-1}$ is not sharp. For more specific John domains we obtain the sharp bound. We say that $D$ satisfies the interior cone condition with aperture $\psi, 0<\psi<\pi / 2$, if for each point $x \in D$ there is a truncated cone with vertex at $x$, aperture $\psi$ and a fixed radius lying in $D$. Obviously, a domain satisfying the interior cone condition with aperture $\psi$ is a John domain with John constant $\sin \psi$.

Theorem 2. Let $D$ be a bounded domain satisfying the interior cone condition with aperture $\psi$ with $\cos \psi>1 / \sqrt{n}$. Then $S^{+}(D) \subset L^{1}(D)$.

For a "slim" John domain we will show $S^{+}(D) \subset L^{p}(D)$ for some $0<p<1$ with the estimate of $p$. This will give a larger $p$ than that in Stegenga-Ullrich [16]. For details see Section 3.

In the previous paper [2], the above theorems are obtained with additional assumption: the capacity density condition (CDC). See [3] for more illustrations. For the 2-dimensional case $\mathrm{CDC}$ is equivalent to the uniform perfectness of the boundary; planar domains bounded by finitely many Jordan curves satisfy CDC. Thus all the known results for $S^{+}(D) \subset L^{p}(D)$ with $p \geq 1$ required CDC or some other stronger exterior condition. The above Theorems 1 and 2 first establish $S^{+}(D) \subset L^{1}(D)$ for a domain satisfying only an interior condition. Recently, Gustafsson, Sakai and Shapiro [9] considered the $L^{1}$-integrability in connection with quadrature domains. They showed that if $D$ is a quadrature domain and the Green functions do not decay so fast near the boundary, then $S^{+}(D) \subset L^{1}(D)$ ([9, Corollary 5.4]).

\section{Proof of the theorems}

For an open set $U$ we denote by $G_{U}$ the Green function for $U$. Throughout this section $D$ is a bounded John domain or a domain satisfying an interior condition. For simplicity we suppress the subscript $D$ and write $G$ for the Green function for $D$. Moreover, $x_{0} \in D$ is a fixed point and let $g(x)=G\left(x, x_{0}\right)$. By the symbol $A$ we denote an absolute positive constant whose value is unimportant and may change 
from line to line. If necessary, we use $A_{1}, A_{2}, \ldots$, to specify them. We shall say that two positive functions $f_{1}$ and $f_{2}$ are comparable, written $f_{1} \approx f_{2}$, if and only if there exists a constant $A \geq 1$ such that $A^{-1} f_{1} \leq f_{2} \leq A f_{1}$. The constant $A$ will be called the constant of comparison.

The proof of the theorems uses the following lower estimate of the Green function. For $0<c_{J}<1$ we let

$$
\alpha_{J}=\log \left[\frac{1-c_{J}}{\left(1+c_{J}\right)^{n-1}}\right] / \log \left(1-c_{J}^{2}\right) .
$$

We observe that $\lim \alpha_{J}=1$ as $c_{J} \rightarrow 1$. Let $c_{n}$ be the solution of the equation $(1+t)^{n+1}(1-t)=1$ for $0<t<1$. Then $\alpha_{J}=2$ for $c_{J}=c_{n}$ and $1<\alpha_{J}<2$ for $c_{n}<c_{J}<1$. We see that $n /(n+2)<c_{n}<1-2^{-n-1}$.

Lemma 1 (see [2, Lemma 12]). (i) If $D$ is a John domain with John constant $c_{J}$, then $g(x) \geq A \delta_{D}(x)^{\alpha_{J}}$.

(ii) If $D$ satisfies the interior cone condition with aperture $\psi$ with $\cos \psi>1 / \sqrt{n}$, then there is $1<\alpha(\psi)<2$ such that $g(x) \geq A \delta_{D}(x)^{\alpha(\psi)}$.

Theorems 1 and 2 readily follow from Lemma 1 and the following.

Theorem 3. Let $D$ be a John domain and suppose $g(x) \geq A \delta_{D}(x)^{\alpha}$ for $\alpha>0$. For $\varepsilon>0$ let $V=(\min \{g, 1\})^{\varepsilon-2 / \alpha}$. Then

$$
\int_{D} u(x) V(x) g(x) d x \leq A u\left(x_{0}\right) \quad \text { for any } u \in S^{+}(D),
$$

where $A$ is independent of $u \in S^{+}(D)$. Moreover, if $0<\alpha<2$, then $S^{+}(D) \subset$ $L^{1}(D)$.

We need one of the main results in [2]. Define the Green capacity $\operatorname{Cap}_{U}(E)$ for $E \subset U$ by

$\operatorname{Cap}_{U}(E)=\sup \left\{\mu(E): G_{U} \mu \leq 1\right.$ on $U, \mu$ is a Borel measure supported on $\left.E\right\}$.

By $B(x, r)$ we denote the open ball with center at $x$ and radius $r$.

Lemma 2 ([2, Theorem 1]). Let $0<\eta<1$. Then for an open set $U$ with Green function $G_{U}$

$$
\sup _{x \in U} \int_{U} G_{U}(x, y) d y \leq A w_{\eta}(U)^{2}
$$

where

$$
w_{\eta}(U)=\inf \left\{\rho>0: \frac{\operatorname{Cap}_{B(x, 2 \rho)}(B(x, \rho) \backslash U)}{\operatorname{Cap}_{B(x, 2 \rho)}(B(x, \rho))} \geq \eta \quad \text { for all } x \in U\right\} .
$$

The above quantity $w_{\eta}(U)$ is called the capacitary width of $U$. The definition of John domain readily implies the following.

Lemma 3. Let $D$ be a John domain. Then $w_{\eta}\left(\left\{x \in D: \delta_{D}(x) \leq r\right\}\right) \leq A r$.

The following estimate of the Green potential is called the basic estimate [4, Theorem 3]. 
Lemma 4. Let $u$ be a positive continuous superharmonic function on D. For an integer $j$ we put $D_{j}=\left\{x \in D: 2^{j-1}<u(x)<2^{j+2}\right\}$ and let $G_{j}$ be the Green function for $D_{j}$. If $f$ is a nonnegative measurable function on $D$, then

$$
\sup _{x \in D} \frac{1}{u(x)} \int_{D} G(x, y) f(y) d y \leq 4 \sum_{j=-\infty}^{\infty} \sup _{x \in D_{j}} \frac{1}{u(x)} \int_{D_{j}} G_{j}(x, y) f(y) d y .
$$

Proof of Theorem 3. Apply Lemma 4 to $u=g$ and $f=V g$ to obtain

$$
\sup _{x \in D} \frac{1}{g(x)} \int_{D} G(x, y) V(y) g(y) d y \leq 32 \sum_{j=-\infty}^{\infty} \sup _{x \in D_{j}} \int_{D_{j}} G_{j}(x, y) V(y) d y,
$$

where $D_{j}=\left\{x \in D: 2^{j-1}<g(x)<2^{j+2}\right\}$. Since $g(x) \geq A \delta_{D}(x)^{\alpha}$, it follows that $D_{j} \subset\left\{x \in D: \delta_{D}(x) \leq A 2^{j / \alpha}\right\}$ and hence from Lemmas 2 and 3 that

$$
\sum_{j=-\infty}^{0} \sup _{x \in D_{j}} \int_{D_{j}} G_{j}(x, y) V(y) d y \leq A \sum_{j=-\infty}^{0}\left(2^{j}\right)^{\varepsilon-2 / \alpha}\left(2^{j / \alpha}\right)^{2} \leq A \sum_{j=-\infty}^{0} 2^{\varepsilon j}<\infty .
$$

On the other hand, if $j \geq 1$, then $D_{j} \subset B\left(x_{0}, A 2^{j /(2-n)}\right)$ if $n \geq 3$ and $D_{j} \subset$ $B\left(x_{0}, \exp \left(-A 2^{j}\right)\right)$ if $n=2$. Hence Lemma 2 implies

$$
\begin{aligned}
\sum_{j=1}^{\infty} \sup _{x \in D_{j}} \int_{D_{j}} G_{j}(x, y) V(y) d y & =\sum_{j=1}^{\infty} \sup _{x \in D_{j}} \int_{D_{j}} G_{j}(x, y) d y \\
& \leq \begin{cases}A \sum_{j=1}^{\infty} 2^{2 j /(2-n)}<\infty & \text { if } n \geq 3, \\
A \sum_{j=1}^{\infty} \exp \left(-A 2^{j}\right)<\infty & \text { if } n=2 .\end{cases}
\end{aligned}
$$

Thus

$$
\int_{D} G(x, y) V(y) g(y) d y \leq A g(x)=A G\left(x, x_{0}\right) .
$$

Integrate the above inequality with respect to $d \mu(x)$ and use Fubini's theorem. Then we have

$$
\int_{D} u(y) V(y) g(y) d y \leq A u\left(x_{0}\right)
$$

with $u=G \mu$. Every $u \in S^{+}(D)$ can be approximated from below by a Green potential, so that the monotone convergence theorem proves the first assertion.

Finally, suppose $0<\alpha<2$. Let $\varepsilon=-1+2 / \alpha>0$ and observe that $V g \geq 1$ and $\int_{D} u d x \leq A u\left(x_{0}\right)$ for $u \in S^{+}(D)$. If $u\left(x_{0}\right)<\infty$, then $u \in L^{1}(D)$ obviously. If $u\left(x_{0}\right)=\infty$, then replace $u$ by its Poisson integral over a small ball with center at $x_{0}$. The replaced function belongs to $S^{+}(D)$ and its value at $x_{0}$ is finite, so that it belongs to $L^{1}(D)$ by the previous observation. This, together with the local integrability of $u$, proves $u \in L^{1}(D)$.

\section{3. $L^{p}$-INTEGRABILITY}

For a bounded John domain with John constant smaller than that in Theorem 1, we shall obtain $L^{p}$-integrability of positive superharmonic functions with $0<p<1$. The exponent $p$ will be estimated in terms of John constant. To this end we show the following lemma, which is inspired by [14, Theorem 4]. 
Lemma 5. Let $D$ be a bounded John domain with John constant $c_{J}$. Then there is a positive constant $\tau_{J}$ depending only on $c_{J}$ and the dimension $n$ such that

$$
\int_{D} \delta_{D}(x)^{-\tau} d x<\infty \quad \text { for } 0<\tau<\tau_{J} .
$$

Here $\tau_{J}$ can be estimated as $\tau_{J} \geq \frac{\log \left(1+\left(c_{J} / 20\right)^{n}\right)}{\log 2}$.

Proof. Let $\widetilde{D}_{j}=\left\{x \in D: 2^{-j-1} \leq \delta_{D}(x)<2^{-j}\right\}$. Then $\bigcup_{j=j_{0}}^{\infty} \widetilde{D}_{j}$ is a disjoint decomposition of $D$ with some $j_{0}$. Observe that $\sum_{j=j_{0}}^{\infty}\left|\widetilde{D}_{j}\right|=|D|<\infty$, where $\left|\widetilde{D}_{j}\right|$ denotes the volume of $\widetilde{D}_{j}$. Without loss of generality we may assume that $j_{0}=0$ and $x_{0} \in D_{0}$. Suppose $x \in \bigcup_{i=j+1}^{\infty} \widetilde{D}_{i}$ with $j \geq 1$, i.e., $\delta_{D}(x)<2^{-j-1}$. By definition there is a rectifiable curve $\gamma$ connecting $x$ and $x_{0}$ with (1.1). We find a point $\xi \in \gamma$ such that $\delta_{D}(\xi)=2^{-j}$. By $(1.1)$

$$
2^{-j}=\delta_{D}(\xi) \geq c_{J} \ell(\gamma(x, \xi)) \geq c_{J}|x-\xi|
$$

so that $|x-\xi| \leq c_{J}^{-1} 2^{-j}$. Hence

$$
\bigcup_{i=j+1}^{\infty} \widetilde{D}_{i} \subset \bigcup_{\delta_{D}(\xi)=2^{-j}} C\left(\xi, c_{J}^{-1} 2^{-j}\right),
$$

where $C\left(\xi, c_{J}^{-1} 2^{-j}\right)$ is the closed ball with center at $\xi$ and radius $c_{J}^{-1} 2^{-j}$. Suppose for a moment $\delta_{D}(\xi)=2^{-j}$. Then, by definition, there is a point $x_{\xi} \in \partial D$ such that $\left|x_{\xi}-\xi\right|=2^{-j}$. Let $\xi^{\prime}$ be the point on the line segment $\overline{x_{\xi} \xi}$ with $\left|\xi-\xi^{\prime}\right|=2^{-j-1}$. Then an elementary geometrical observation shows that $\delta_{D}\left(\xi^{\prime}\right)=\frac{1}{2}\left(2^{-j}+2^{-j-1}\right)$ and $B\left(\xi^{\prime}, 2^{-j-2}\right) \subset \widetilde{D}_{j}$, so that

$$
\left|\widetilde{D}_{j} \cap C\left(\xi, c_{J}^{-1} 2^{-j}\right)\right| \geq A_{0}\left(2^{-j-2}\right)^{n}=\left(\frac{c_{J}}{20}\right)^{n}\left|C\left(\xi, 5 c_{J}^{-1} 2^{-j}\right)\right|,
$$

where $A_{0}$ is the volume of a unit ball. By the covering lemma (see e.g. [17, Theorem 1.3.1]) we can find $\xi_{k}$ such that $\delta_{D}\left(\xi_{k}\right)=2^{-j},\left\{C\left(\xi_{k}, c_{J}^{-1} 2^{-j}\right)\right\}_{k}$ is disjoint and

$$
\bigcup_{i=j+1}^{\infty} \widetilde{D}_{i} \subset \bigcup_{k} C\left(\xi_{k}, 5 c_{J}^{-1} 2^{-j}\right)
$$

In view of (3.1) we have

$$
\sum_{i=j+1}^{\infty}\left|\widetilde{D}_{i}\right| \leq \sum_{k}\left|C\left(\xi_{k}, 5 c_{J}^{-1} 2^{-j}\right)\right| \leq\left(\frac{20}{c_{J}}\right)^{n} \sum_{k}\left|\widetilde{D}_{j} \cap C\left(\xi_{k}, c_{J}^{-1} 2^{-j}\right)\right| \leq\left(\frac{20}{c_{J}}\right)^{n}\left|\widetilde{D}_{j}\right| .
$$

Multiply the above inequalities by $r^{j}$ and take the summation for $j=1, \ldots, N-1$, where $r>1$ is a constant to be determined. Then

$$
\begin{aligned}
\left(\frac{20}{c_{J}}\right)^{n} \sum_{j=1}^{N-1} r^{j}\left|\widetilde{D}_{j}\right| & \geq \sum_{j=1}^{N-1} \sum_{i=j+1}^{\infty} r^{j}\left|\widetilde{D}_{i}\right|=\sum_{i=2}^{\infty} \sum_{j=1}^{\min \{N, i\}-1} r^{j}\left|\widetilde{D}_{i}\right| \\
& \geq \sum_{i=2}^{N-1} \sum_{j=1}^{i-1} r^{j}\left|\widetilde{D}_{i}\right|=\sum_{i=2}^{N-1} \frac{r^{i}-r}{r-1}\left|\widetilde{D}_{i}\right| \\
& =\frac{1}{r-1} \sum_{i=1}^{N-1} r^{i}\left|\widetilde{D}_{i}\right|-\frac{r}{r-1} \sum_{i=1}^{N-1}\left|\widetilde{D}_{i}\right|,
\end{aligned}
$$


so that

$$
\frac{r}{r-1} \sum_{i=1}^{N-1}\left|\widetilde{D}_{i}\right| \geq\left(\frac{1}{r-1}-\left(\frac{20}{c_{J}}\right)^{n}\right) \sum_{i=1}^{N-1} r^{i}\left|\widetilde{D}_{i}\right| .
$$

Letting $N \rightarrow \infty$, we obtain

$$
\infty>\frac{r}{r-1}|D|=\frac{r}{r-1} \sum_{i=1}^{\infty}\left|\widetilde{D}_{i}\right| \geq\left(\frac{1}{r-1}-\left(\frac{20}{c_{J}}\right)^{n}\right) \sum_{i=1}^{\infty} r^{i}\left|\widetilde{D}_{i}\right| .
$$

Let $1<r<1+\left(c_{J} / 20\right)^{n}$. Then

$$
\left(\frac{1}{r-1}-\left(\frac{20}{c_{J}}\right)^{n}\right)>0
$$

and the above inequality implies

$$
\sum_{i=1}^{\infty} r^{i}\left|\widetilde{D}_{i}\right|<\infty
$$

We observe that

$$
r^{i} \approx \delta_{D}(x)^{-\log r / \log 2} \quad \text { for } x \in \widetilde{D}_{i},
$$

whence

$$
\int_{D} \delta_{D}(x)^{-\log r / \log 2} d x<\infty
$$

This proves the lemma.

Theorem 4. Let $D$ be a bounded John domain with John constant $c_{J}$. Suppose $g(x) \geq A \delta_{D}(x)^{\alpha}$ for $x \in D$ with $\alpha \geq 2$ and

$$
\int_{D} \delta_{D}(x)^{-\tau} d x<\infty
$$

with $\tau>0$. Then $S^{+}(D) \subset L^{p}(D)$ for $0<p<\tau /(\alpha-2+\tau)$.

Remark. Let $\alpha_{J}$ be as in (2.1) and let $\tau_{J}$ be as in Lemma 5. If $\alpha_{J} \geq 2$, then Lemmas 1 and 5 show that $S^{+}(D) \subset L^{p}(D)$ with $0<p<p_{J}=\tau_{J} /\left(\alpha_{J}-2+\tau_{J}\right)$. Observe that $p_{J} \approx c_{J}^{n+1}$ as $c_{J} \rightarrow 0 ; p_{J} \rightarrow 1$ as $c_{J} \rightarrow c_{n}$.

Proof. Let $0<p<\tau /(\alpha-2+\tau)$. Put

$$
\varepsilon=\frac{1}{\alpha}\left(\frac{(1-p) \tau}{p}-\alpha+2\right) .
$$

Then $\varepsilon>0$ and $\alpha(\varepsilon-2 / \alpha+1) p /(1-p)=\tau$. Let $D^{\prime}=\{x \in D: g(x) \leq 1\}$. Take $u \in S^{+}(D)$. Then Hölder's inequality and Theorem 3 yield

$$
\begin{aligned}
\int_{D^{\prime}} u^{p} d x & \leq\left(\int_{D^{\prime}} u g^{\varepsilon-2 / \alpha+1} d x\right)^{p}\left(\int_{D^{\prime}} g^{-(\varepsilon-2 / \alpha+1) p /(1-p)} d x\right)^{1-p} \\
& \leq A u\left(x_{0}\right)^{p}\left(\int_{D^{\prime}} \delta_{D}^{-\tau} d x\right)^{1-p} \leq A u\left(x_{0}\right)^{p}
\end{aligned}
$$

where (3.2) is used in the last inequality. By the same reasoning as in the proof of Theorem 3, we have $\int_{D^{\prime}} u^{p} d x<\infty$. This, together with the local integrability of a superharmonic function, proves the theorem. 


\section{REFERENCES}

[1] H. Aikawa, Integrability of superharmonic functions and subharmonic functions, Proc. Amer. Math. Soc. 120 (1994), 109-117. MR 94b:31003

[2] H. Aikawa, Norm estimate of Green operator, perturbation of Green function and integrability of superharmonic functions, Math. Ann. 312 (1998), 289-318. CMP 99:08

[3] H. Aikawa, Norm estimate for the Green operator with applications, Proceedings of Complex Analysis and Differential Equations, Marcus Wallenberg Symposium in honor of Matts Essén, Uppsala University (1997) (to appear).

[4] H. Aikawa and M. Murata, Generalized Cranston-McConnell inequalities and Martin boundaries of unbounded domains, J. Analyse Math. 69 (1996), 137-152. MR 98f:31016

[5] D. H. Armitage, On the global integrability of superharmonic functions in balls, J. London Math. Soc. (2) 4 (1971), 365-373.

[6] D. H. Armitage, Further result on the global integrability of superharmonic functions, J. London Math. Soc. (2) 6 (1972), 109-121. MR 42:2078

[7] F. W. Gehring and O. Martio, Lipschitz classes and quasiconformally homogeneous domains, Ann. Acad. Sci. Fenn. 10 (1985), 203-219. MR 87b:30029

[8] Y. Gotoh, Integrability of superharmonic functions, uniform domains, and Hölder domains, Proc. Amer. Math. Soc. (to appear). CMP 98:03

[9] B. Gustafsson, M. Sakai and H. S. Shapiro, On domains in which harmonic functions satisfy generalized mean value properties, Potential Analysis 7 (1997), 467-484. CMP 97:16

[10] P. Lindqvist, Global integrability and degenerate quasilinear elliptic equations, J. Analyse Math. 61 (1993), 283-292. MR 95b:35062

[11] F.-Y. Maeda and N. Suzuki, The integrability of superharmonic functions on Lipschitz domains, Bull. London Math. Soc. 21 (1989), 270-278. MR 90b:31004

[12] M. Masumoto, A distortion theorem for conformal mappings with an application to subharmonic functions, Hiroshima Math. J. 20 (1990), 341-350. MR 91h:30031

[13] M. Masumoto, Integrability of superharmonic functions on plane domains, J. London Math. Soc. (2) 45 (1992), 62-78. MR 93f:31002

[14] W. Smith and D. A. Stegenga, Hölder domains and Poincaré domains, Trans. Amer. Math. Soc. 319 (1990), 67-100. MR 90i:30012

[15] W. Smith and D. A. Stegenga, Exponential integrability of the quasi-hyperbolic metric on Hölder domains, Ann. Acad. Sci. Fenn. 16 (1991), 345-360. MR 93b:30016

[16] D. A. Stegenga and D. C. Ullrich, Superharmonic functions in Hölder domains, Rocky Mountain J. Math. 25 (1995), 1539-1556. MR 97j:31003

[17] W. P. Ziemer, Weakly Differentiable Functions, Springer-Verlag, 1989. MR 91e:46046

Department of Mathematics, Shimane University, Matsue 690-8504, Japan

E-mail address: haikawa@math.shimane-u.ac.jp 